SUBJECT AREAS:

OPTICAL MANIPULATION

AND TWEEZERS

STATISTICAL PHYSICS

Received

27 September 2013

Accepted

14 January 2014

Published

5 February 2014

Correspondence and requests for materials should be addressed to G.V. Igiorgio.volpe@ espci.fr)

\title{
Brownian Motion in a Speckle Light Field: Tunable Anomalous Diffusion and Selective Optical Manipulation
}

\author{
Giorgio Volpe', Giovanni Volpe ${ }^{2}$ \& Sylvain Gigan'
}

\author{
'Institut Langevin, UMR7587 of CNRS and ESPCI ParisTech, 1 rue Jussieu, 75005 Paris, France, ${ }^{2}$ Physics Department, Bilkent \\ University, Çankaya, 06800 Ankara, Turkey.
}

The motion of particles in random potentials occurs in several natural phenomena ranging from the mobility of organelles within a biological cell to the diffusion of stars within a galaxy. A Brownian particle moving in the random optical potential associated to a speckle pattern, i.e., a complex interference pattern generated by the scattering of coherent light by a random medium, provides an ideal model system to study such phenomena. Here, we derive a theory for the motion of a Brownian particle in a speckle field and, in particular, we identify its universal characteristic timescale. Based on this theoretical insight, we show how speckle light fields can be used to control the anomalous diffusion of a Brownian particle and to perform some basic optical manipulation tasks such as guiding and sorting. Our results might broaden the perspectives of optical manipulation for real-life applications.

$\mathrm{V}$ arious phenomena rely on particles performing stochastic motion in random potentials. Examples range from the nanoscopic world of molecules undergoing anomalous diffusion within the cytoplasm of a cell ${ }^{1}$ to the Brownian motion of stars within galaxies ${ }^{2}$. Another example of this kind of phenomena is given by the motion of a Brownian particle in a random optical potential generated by a speckle pattern, i.e., the random light field resulting from complex light scattering in optically complex media, such as biological tissues, turbid liquids and rough surfaces (see background in Figure 1a-c) ) $^{3,4}$. This latter example is particularly suited to work as a model system because its parameters (e.g., particle size and material, illumination light) are easily controllable and its dynamics are easily accessible by standard optical microscopy techniques ${ }^{5}$. Earlier experimental works showed the possibility of trapping particles in high-intensity speckle light fields $s^{6-9}$, the simplest optical manipulation task, and the emergence of superdiffusion in an active media constituted by a dense solution of microparticles that generates a time-varying speckle field ${ }^{10}$. However, apart from these previous studies, there is little understanding of the interaction of Brownian motion with random light potentials and the intrinsic randomness of speckle patterns is largely considered a nuisance to be minimized for most purposes, e.g., in optical manipulation ${ }^{11,12}$. In fact, similar and even more complex effects have been extensively studied using periodic potentials rather than random potentials: these studies include the observation of giant diffusion induced by an oscillating periodic potential ${ }^{13}$, and the demonstration of guiding and sorting particles using either moving periodic potentials $\mathrm{s}^{14-16}$ or static periodic potentials in microfluidic flows ${ }^{17-19}$. It is not a priori obvious that the same phenomena that have been observed with periodic potentials can also arise with random potentials, as the statistical properties of random potentials fundamentally differ from those of periodic potentials. For example, in non-equilibrium statistical physics, the dynamics of a Brownian particle in a moving periodic potential can be described as a straightforward generalization of the dynamics of a Brownian particle in static not out-of-equilibrium potentials, while this is no longer the case for random potentials for which a full out-of-equilibrium description is still required ${ }^{20}$.

In this Letter, we study the Brownian motion of a particle in a speckle pattern and, in particular, we derive the characteristic timescale $\tau$ of such motion, which is universal as it depends only on the universal properties of speckle light fields ${ }^{3,21,22}$. This theoretical insight permits us to demonstrate that random potentials can also be used instead of more regular potentials to control the dynamics of a Brownian particle in a very accurate way, despite their intrinsic randomness. First of all, while the emergence of superdiffusion in time-varying speckle patterns has already been observed ${ }^{10}$, we demonstrate that speckle fields provide a tunable and controllable model system to study anomalous diffusion by relating $\tau$ to the emergence of subdiffusion and superdiffusion in time-varying speckle patterns. Moreover, we demonstrate the possibility of harnessing the memory effect of speckle field ${ }^{21,22}$ to 


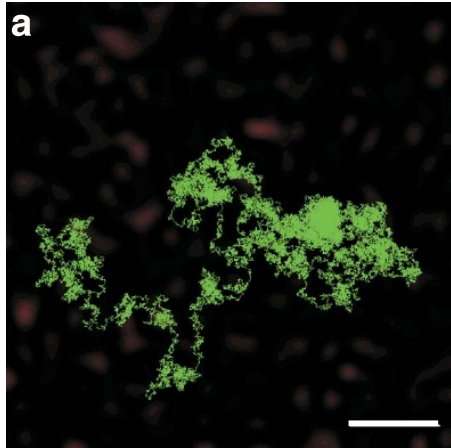

$\langle F\rangle=10 \mathrm{fN}$

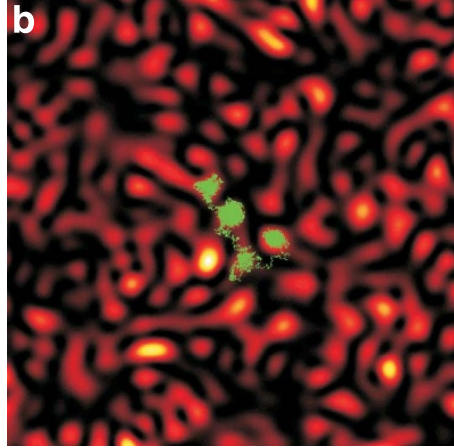

$\langle F\rangle=50 \mathrm{fN}$

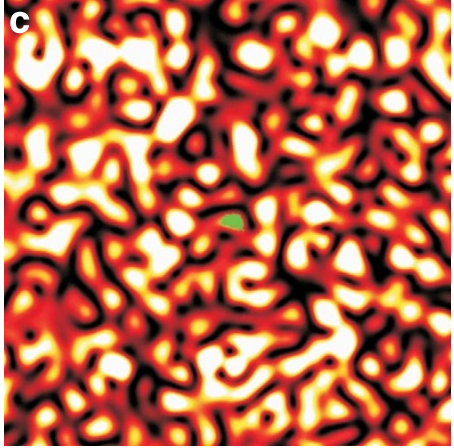

$\langle F\rangle=200 \mathrm{fN}$
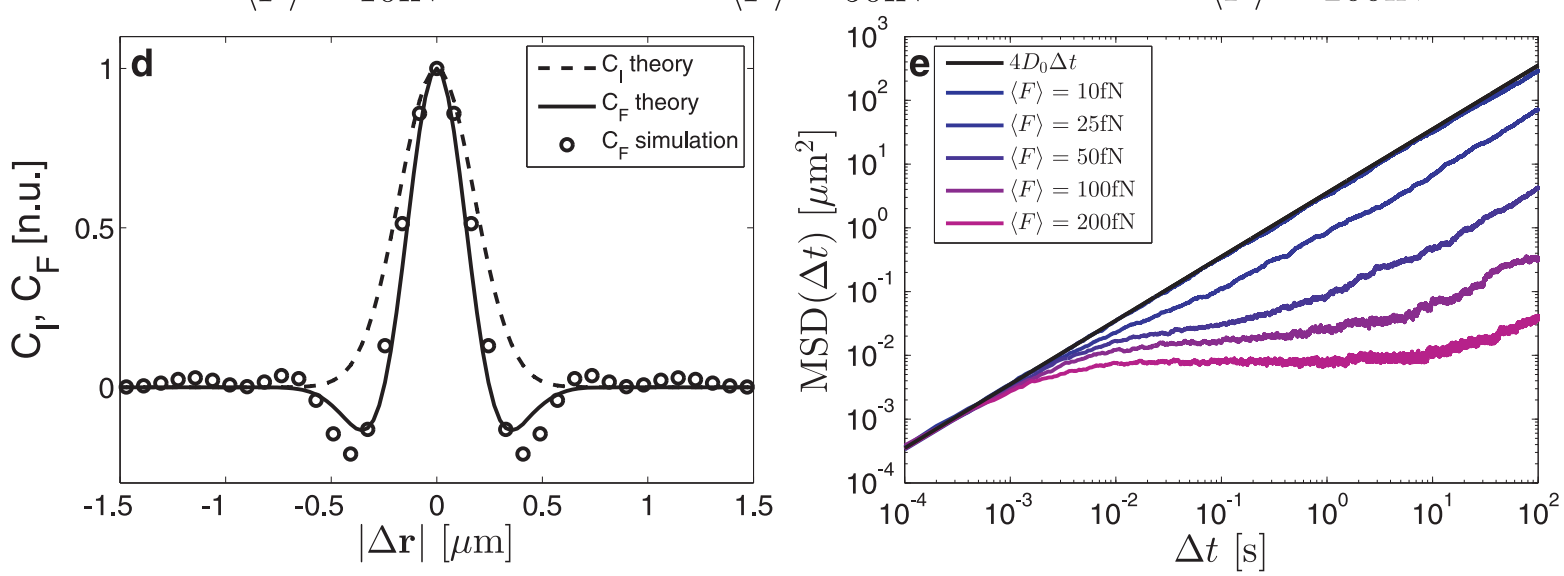

Figure $1 \mid$ Subdiffusion in a static speckle pattern. (a-c) The background represents a speckle pattern generated by a circular aperture $(\lambda=1064 \mathrm{~nm}$, speckle grain $490 \mathrm{~nm}$ ); the white scale bar corresponds to $2 \mu \mathrm{m}$. The trajectories (green solid lines) show progressive confinement of a polystyrene bead $\left(\mathrm{R}=250 \mathrm{~nm}, n_{p}=1.59\right)$ in water $\left(n_{m}=1.33, \eta=0.001 \mathrm{Ns} / \mathrm{m}^{2}, \mathrm{~T}=300 \mathrm{~K}\right)$ as a function of the increasing speckle intensity corresponding to an average force on the particle of $(\mathrm{a})\langle\mathrm{F}\rangle=10 \mathrm{fN}\left(\langle\mathrm{I}\rangle=13 \mathrm{~mW} / \mu \mathrm{m}^{2}\right),(\mathrm{b})\langle\mathrm{F}\rangle=50 \mathrm{fN}\left(\langle\mathrm{I}\rangle=65 \mathrm{~mW} / \mu \mathrm{m}^{2}\right),(\mathrm{c})\langle\mathrm{F}\rangle=200 \mathrm{fN}\left(\langle\mathrm{I}\rangle=260 \mathrm{~mW} / \mu \mathrm{m}^{2}\right) .(\mathrm{d}) \mathrm{Normalized}$ autocorrelation function of the force field produced by the speckle pattern according to our theoretical model (solid line) and in the simulated speckle pattern (circles). The dashed line represents the theoretical normalized autocorrelation function of the speckle pattern intensity. (e) Brownian particle mean square displacements as a function of $\langle\mathrm{F}\rangle$ (purple lines), and their deviation from Einstein's free diffusion law (black line).

perform basic optical manipulation tasks such as guiding and sorting $^{23}$, which go beyond selective optical trapping in high-intensity speckle grains ${ }^{7,8}$.

\section{Results}

Speckle light patterns can be generated by different processes, such as scattering of a laser on a rough surface, multiple scattering in an optically complex medium, or mode-mixing in a multimode fiber ${ }^{4}$. In general, they are the result of the interference of a large number of waves propagating along different directions and with a random phase distribution, and, despite their random appearance, they share some universal statistical properties ${ }^{3,24}$ (see Supplementary Note S1). In particular, a speckle pattern has a negative exponential intensity distribution, and the normalized spatial autocorrelation function $C_{I}(\Delta \mathbf{r})$ can be approximated by a Gaussian ${ }^{25}$ :

$$
C_{I}(\Delta \mathbf{r})=\frac{\langle I(\mathbf{r}+\Delta \mathbf{r}) I(\mathbf{r})\rangle}{\left\langle I(\mathbf{r})^{2}\right\rangle} \approx e^{-\frac{|\Delta \mathbf{r}|^{2}}{2 \sigma^{2}}},
$$

where $I(\mathbf{r})$ is the speckle pattern intensity as a function of the position $\mathbf{r}$ and the standard deviation $\sigma \approx d / 3$ is proportional to the average speckle grain size $d$ (see Supplementary Note S1).

The motion of a Brownian particle in a static speckle field is the result of random thermal forces and deterministic optical forces. In the following discussion, we focus on particles whose radius is smaller than the light wavelength $\lambda$. We develop a theory that is strictly valid for Rayleigh particles (see Methods), but our conclusions hold also for larger particles ${ }^{26}$. In particular, as we show in the
Supplementary Figure S1 using exact electromagnetic theory ${ }^{27,28}$, our theory is also approximately valid for bigger particles provided that their radius is smaller than the light wavelength $\lambda$. Optical gradient forces are the dominant deterministic forces acting on small particles, and they attract high-refractive index particles towards the intensity maxima of the optical field ${ }^{26}$.

As a particle moves in a speckle field, the optical force acting on it changes both in magnitude and direction with a characteristic time scale $\tau=\frac{L}{<\mathrm{v}\rangle}$, where $L$ is the correlation length of the optical force field and $\langle\mathrm{v}\rangle$ is the average particle drift speed. The optical force field correlation function (Figure 1d) is

$$
C_{F}(\Delta \mathbf{r})=\frac{\kappa^{2}}{\sigma^{2}}\langle I\rangle^{2}\left(2-\frac{|\Delta \mathbf{r}|^{2}}{\sigma^{2}}\right) e^{-\frac{\mid \Delta r^{2}}{2 \sigma^{2}}},
$$

where $\kappa=\frac{1}{4} \operatorname{Re}(\alpha)$ and $\alpha$ is the particle polarizability, so that $L=\sqrt{2} \sigma$ (see detailed derivation in Methods and Supplementary Note S2). Since the particle motion is overdamped ${ }^{29}$, the average particle drift speed is $\langle\mathrm{v}\rangle=\frac{\langle F\rangle}{\gamma}$, where $\gamma$ is the particle friction coefficient and $\langle F\rangle \approx \frac{\kappa}{\sigma}\langle I\rangle$ is the average force (see Supplementary Note S2). Thus, we obtain

$$
\tau \approx \sqrt{2} \frac{\sigma^{2} \gamma}{\kappa\langle I\rangle}
$$


which is our central theoretical result and permits us to demonstrate how random optical fields can also be used instead of more regular ones to control the dynamics of a Brownian particle, despite the intrinsic randomness of the field. In the following, after considering how $\tau$ is related to the Brownian motion of a particle in a static speckle pattern, we will present two such examples via numerical experiments.

We start by considering the motion of a particle in a static speckle pattern. As shown by the trajectory in Figure 1a, when the optical forces are relatively low (average force $\langle F\rangle=10 \mathrm{fN}$ ), the particle is virtually freely diffusing. As the forces increase (Figure $1 \mathrm{~b},\langle F\rangle=$ $50 \mathrm{fN}$ ), first a subdiffusive behavior emerges where the particle is metastably trapped in the speckle grains, while it can still move between them ${ }^{30}$. Finally, for even stronger forces (Figure $1 \mathrm{c},\langle F\rangle=$ $200 \mathrm{fN}$ ), the particle remains trapped in one of the speckle grains for a very long time, as previously observed experimentally ${ }^{6-9}$. These observations can be interpreted in terms of $\tau$ : for relatively high forces $(\langle F\rangle=200 \mathrm{fN}, \tau \approx 5.6 \mathrm{~ms}), \tau$ is quite low which means that the particle, on average, experiences a restoring force towards its previous position quite soon in its motion, having little possibility to escape a speckle grain; for relatively low forces $(\langle F\rangle=10 \mathrm{fN}, \tau \approx$ $112.5 \mathrm{~ms}$ ) instead, $\tau$ is much higher which means that the particle has time to diffuse away from a speckle grain before actually experiencing the influence of the optical forces exerted by it. These qualitative considerations can be made more precise by calculating the mean square displacement $\operatorname{MSD}(\Delta t)$ of the particle motion (see Methods). As shown in Figure 1e, for low optical forces and high $\tau$ the mean square displacement is substantially linear in $\Delta \mathrm{t}$, i.e. $\operatorname{MSD}(\Delta t) \approx$ $4 D_{S E} \Delta t$, where $D_{S E}$ is the Stokes-Einstein diffusion coefficient. As the forces increase and $\tau$ decreases, there is a transition towards a subdiffusive regime characterized by $\operatorname{MSD}(\Delta t) \propto \Delta t^{\beta}$ with $\beta<1$. We remark that, for very large $\Delta t$, the motion returns diffusive, i.e. $\beta$ $=1$, albeit with an effective diffusion coefficient $D_{\text {eff }}<D_{S E}$. Interestingly, in many naturally occurring anomalous diffusion processes, such as the subdiffusion of molecules within the living cell, the distribution of waiting times is expected to correspond to a random walker continually caught in potential wells whose depths are distributed exponentially ${ }^{1}$. Compared to periodic potentials, therefore, speckle patterns represent better model systems to study such phenomena: in fact, while periodic potentials are characterized by only a few potential depths, the distribution of potential depths (e.g. the distribution of intensities) in a speckle pattern follows a negative exponential distribution ${ }^{3,24}$.
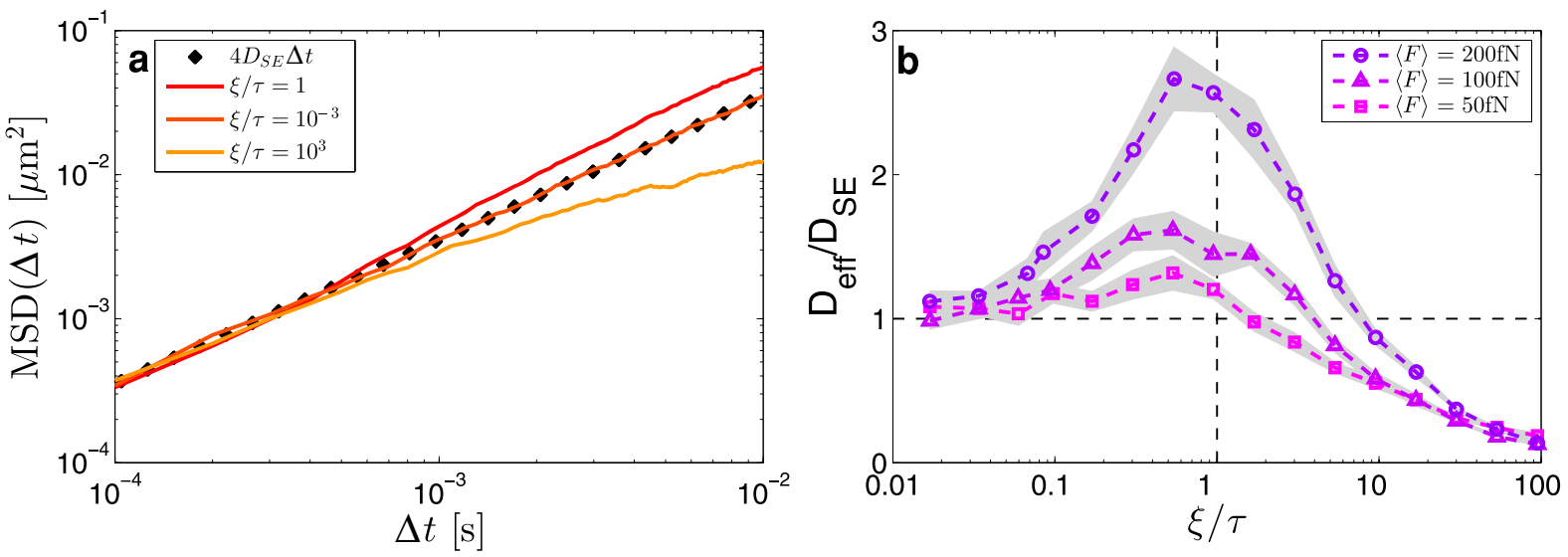

Figure $2 \mid$ Superdiffusion in a time varying speckle pattern. (a) Mean square displacements in logarithmic scale for a Brownian particle moving in a speckle pattern which varies on a timescale $\xi \approx \tau$ (red line), $\xi \ll \tau$ (orange line), and $\xi \gg \tau$ (yellow line). The dots represent Einstein's free diffusion law. (b) The effective diffusion of the motion at long timescales as a function of $\xi / \tau$ shows a transition from subdiffusion $\left(D_{\text {eff }}<D_{S E}\right)$ to superdiffusion $\left(D_{\text {eff }}\right\rangle$ $\left.D_{S E}\right)$. The maximum value of the superdiffusion appears for $\xi \approx \tau(\tau \approx 22.5 \mathrm{~ms}$ for $\langle\mathrm{F}\rangle=50 \mathrm{fN}, \tau \approx 11.2 \mathrm{~ms}$ for $\langle\mathrm{F}\rangle=100 \mathrm{fN}$ and $\tau \approx 5.6 \mathrm{~ms}$ for $\langle\mathrm{F}\rangle=200 \mathrm{fN})$. Every mean point is averaged over 500 particle trajectories $100 \mathrm{~s}$ long, and whose initial position was randomly chosen within the speckle field. The gray shaded areas represent one standard deviation around the average values. 
$V_{\mathrm{S}} /\langle v\rangle \approx 1$, as shown in Figure 3a. A small speckle pattern translation up to a few micrometers can be implemented capitalizing on the speckle property known as memory effect ${ }^{21,22}$ : for a speckle pattern generated by a thin sample, a small tilt of the illumination, easily achievable, e.g., with a galvanometric mirror or an acousto-optic deflector, entails a small spatial translation of the speckle pattern. As shown in Figure $3 \mathrm{~b}$, this is sufficient to realize a Brownian ratchet $^{33}$ : the speckle pattern repeatedly shifts first slowly $\left(V_{\mathrm{S}} /\langle v\rangle=\right.$ 1) by $1 \mu \mathrm{m}$ in the positive direction, which exerts a strong drag on the particle, and then fast $\left(V_{\mathrm{S}} /\langle v\rangle=10\right)$ back to the initial position, which has little effect on the particle position. In $250 \mathrm{~ms}$, the particle is dragged by $\approx 3 \mu \mathrm{m}$ in the direction of the speckle pattern shift, while the particle's trajectory in the perpendicular direction remains unaffected (Figure 3c).

This guiding capability of a speckle pattern can be combined with standard microfluidic systems in order to perform tasks, such as sieving and sorting. For example, a static speckle pattern can be employed to realize a speckle sieve (Figure 4a and Supplementary Video S1). As a liquid containing $200 \mathrm{~nm}$ and $250 \mathrm{~nm}$ particles flows from left to right at $42 \mu \mathrm{m} / \mathrm{s}$, a static speckle pattern efficiently holds the larger particles back while the smaller ones go through almost unaffected; interestingly, the size of the particle that are held back can be dynamically adjusted by changing the intensity of the speckle pattern. A spatiotemporal varying speckle field instead can be employed to realize a speckle sorter (Figure $4 \mathrm{~b}$ and Supplementary Video S2). In a configuration similar to the one for the speckle sieve, the memory effect can be used to exert a perpendicular force to the flow selectively on the larger particles, so that each kind of particle gets guided into a different channel.

\section{Discussion}

Using similar configurations, which are routinely used in microfluiduics, it is possible to separate particles on the basis of various parameters, such as their size or their refractive index, as shown in Supplementary Figures S2 and S3; the resolution of this optical fractionation $^{17-19}$ is only limited by the size of the speckle field, i.e., the longer the speckle field the higher the sensitivity in particle's size or refractive index. These devices can, therefore, be scaled to achieve the high throughput or sensitivity needed in microfluidics (thousands of particles per second) by increasing the flow speed and laser power, as it is also the case for alternative optofluidics devices ${ }^{17-19}$. Moreover, an additional advantage of speckle patterns is that they are also intrinsically widefield, and could be used to sort many particles in parallel in a broader microfluidic chamber, where flow speed is strongly reduced. From the experimental point of view, speckle light fields with such universal statistical properties are straightforwardly generated over large areas using different processes, such as scattering of a laser on a rough surface, multiple scattering in an optically complex medium, or mode-mixing in a multimode fiber ${ }^{4}$. In the Supplementary Note S3, we propose some possible setups that can be implemented at low cost, with little alignment and few optical components based on these processes of generation of a speckle field. Here, we have mainly considered possible experimental implementations where the Brownian particles are moving in a quasi 2-dimensional speckle pattern. This is
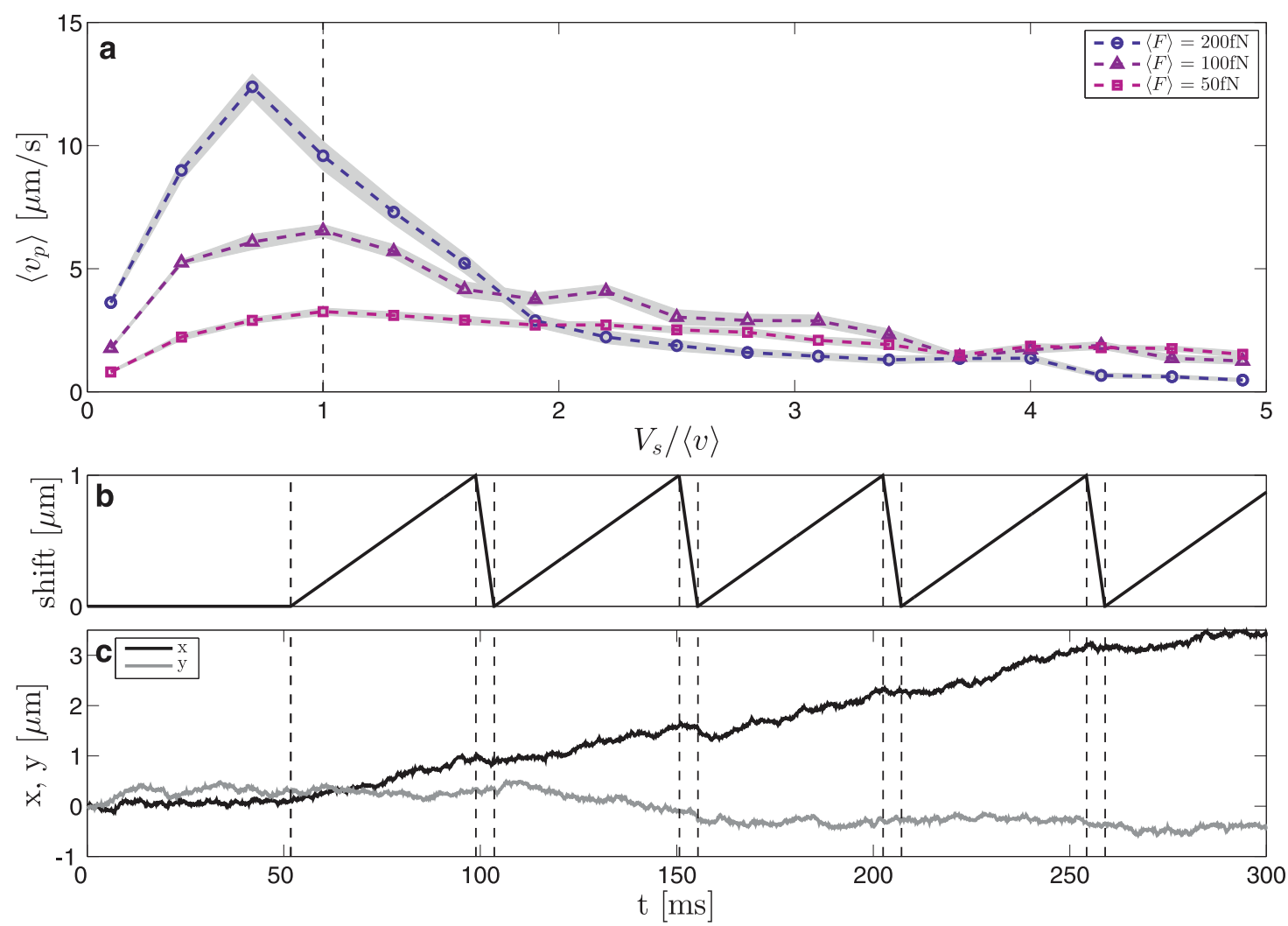

Figure $3 \mid$ Guiding by the speckle memory effect. (a) Average guiding velocity $\left\langle v_{p}\right\rangle$ in the direction of the speckle pattern shift as a function of the shift speed $V_{\mathrm{S}}$ for $\langle\mathrm{F}\rangle=200 \mathrm{fN}$ (circles), $\langle\mathrm{F}\rangle=100 \mathrm{fN}$ (triangles), and $\langle\mathrm{F}\rangle=50 \mathrm{fN}$ (squares). The maximum $\left\langle v_{p}\right\rangle$ is achieved for $V_{\mathrm{S}} \approx\langle v\rangle(\langle v\rangle \approx 10.5 \mu \mathrm{m} / \mathrm{s}$ for $\langle\mathrm{F}\rangle=50 \mathrm{fN},\langle v\rangle \approx 21.2 \mu \mathrm{m} / \mathrm{s}$ for $\langle\mathrm{F}\rangle=100 \mathrm{fN}$ and $\langle v\rangle \approx 42.4 \mu \mathrm{m} / \mathrm{s}$ for $\langle\mathrm{F}\rangle=200 \mathrm{fN})$. Every mean velocity point is calculated over 500 trajectories simulated during $10 \mathrm{~s}$ and whose initial position was randomly chosen within the speckle field. The gray shaded areas represent one standard deviation around the average values. (b) Speckle pattern shift and (c) particle displacement as a function of time in the direction of the speckle pattern shift $x$ (black line) and in the orthogonal direction $y$ (gray line). The speckle pattern repeatedly shifts first slowly in the positive direction by $1 \mu \mathrm{m}$, and then fast to the initial position. The dashed lines represent the instant of time when there is a change of trend in the speckle pattern shift. 

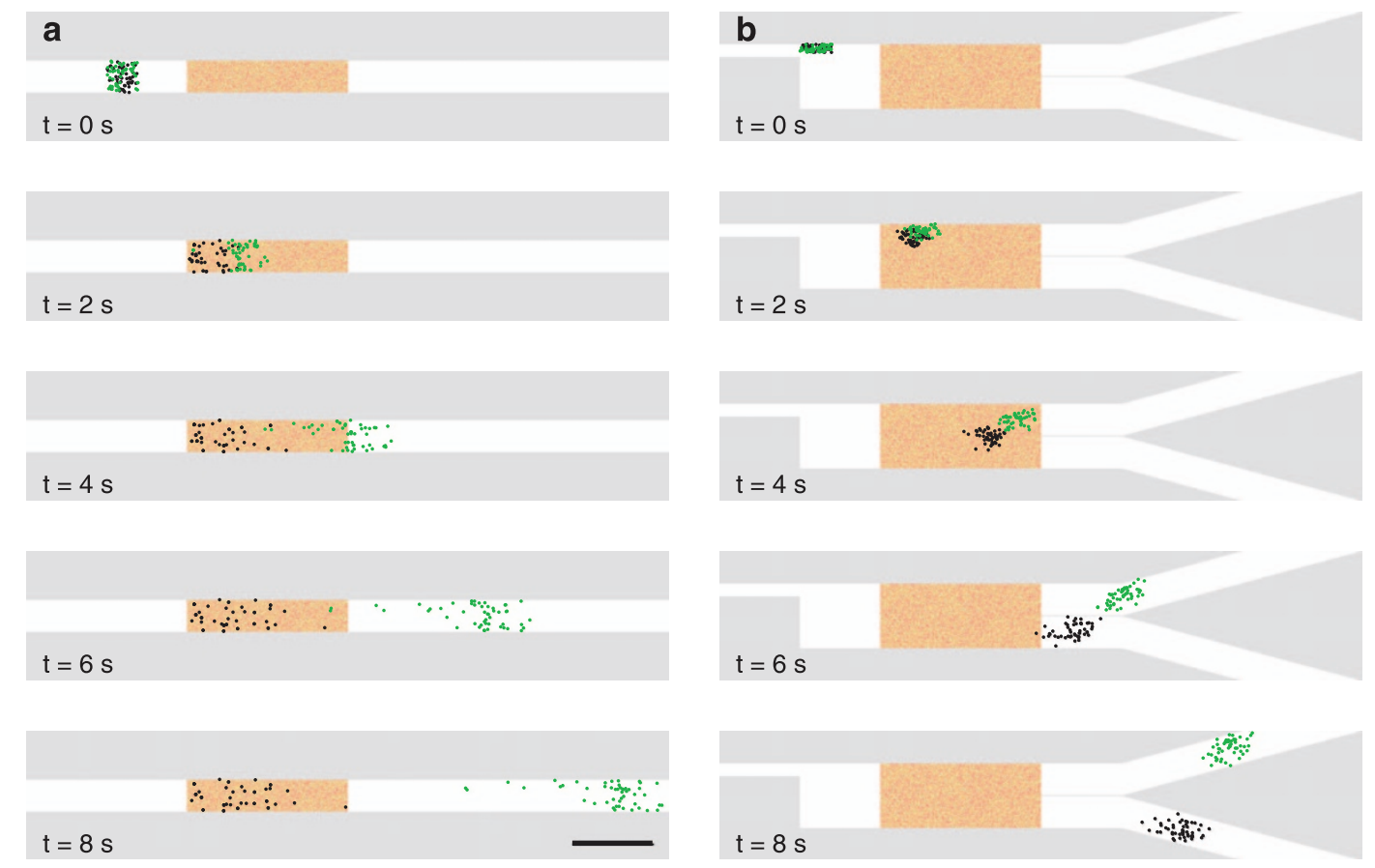

Figure $4 \mid$ Microfluidic speckle sieve and speckle sorter. (a) Lapse-time snapshots of the motion of polystyrene particles with radius $\mathrm{R}=200 \mathrm{~nm}(\langle F\rangle=$ $90 \mathrm{fN}$, green dots) and $\mathrm{R}=250 \mathrm{~nm}(\langle F\rangle=46 \mathrm{fN}$, black dots) in a microfluidic speckle sieve, where a static speckle pattern (red shaded area) traps the smaller particles while it lets the larger particles go away with the flow (flow speed $42 \mu \mathrm{m} / \mathrm{s}$, see also Supplementary Video S1). (b) Same as in (a), but with the speckle pattern ratcheting in the direction orthogonal to the flow by $1 \mu \mathrm{m}$ (flow speed $34 \mu \mathrm{m} / \mathrm{s}$, see also Supplementary Video S2). The black scale bar represents $50 \mu \mathrm{m}$.

particularly true for experiments that are designed satisfying one of the following conditions: the speckle field is generated with a low numerical aperture so that the average longitudinal extension of the speckle grain extends over the depth of the microfluidic channel; optical scattering forces push the particles in the direction of propagation of light, so that in the presence of a boundary, such as the glass or PDMS surface of a microchannel, they effectively confine the particles in a quasi two dimensional space ${ }^{34}$; or the motion of the particles is confined to a quasi 2-dimensional volume far from any physical boundary, for example, by employing acoustic standing waves ${ }^{35}$. In more complex settings, our results can be readily extended to take into account a 3-dimensional description of the speckle field ${ }^{24}$. Particular care should be paid to extent the speckle memory effect to a 3-dimensional case: while the standard memory effect achieves a lateral translation of the speckle field by adding a linear phase gradient at the input of a complex medium ${ }^{21,22}$, a longitudinal translation of the speckle field can be obtained by adding a quadratic phase gradient at the input ${ }^{36}$. Therefore a combination of these two effects allows one to translate the results of our work to a 3-dimensional situation.

In conclusion, we have developed a theoretical framework that allows one to convert the randomness of a speckle light field into a tunable tool to influence the motion of a Brownian particle. In particular, we performed numerical experiments to show the applicability of this concept to the tunable control of anomalous diffusion and to perform standard optical manipulation tasks, such as sorting and guiding. While some of the tasks we propose in the manuscript can be achieved using optical traps or lattices in periodic arrangements ${ }^{13-19}$, the alternative use of random potentials offers some additional advantages, such as intrinsic robustness to noise and aberrations from the optics and the environment. In fact, the use of random optical potentials over periodic ones has the advantage of requiring very simple optical setups as well as a very low degree of control over the experimental environment, thus offering the possibility of performing optical manipulation through scattering media, such as diffusers and biological tissue, where light propagation naturally leads to the formation of speckle patterns, without recurring to wavefront shaping ${ }^{11}$.

\section{Methods}

Simulation of Brownian motion in a speckle pattern. The motion of a Brownian particle of radius $R$ in a generic force field can be modeled with the following Langevin equation $^{37}$ :

$$
m \ddot{\mathbf{r}}+\gamma \dot{\mathbf{r}}=\sqrt{2 k_{B} T \gamma} \mathbf{W}+\mathbf{F}(\mathbf{r}),
$$

where $\dot{\mathbf{r}}, m$ and $\gamma=6 \pi \eta R$ are respectively the particle's velocity, mass and friction coefficient, $\eta$ the viscosity of the surrounding medium, $\mathrm{W}$ a white noise vector, $k_{B}$ the Boltzmann constant and $T$ the temperature of the system. For a Rayleigh particle, $F(\mathbf{r})$ $=\kappa \nabla I(\mathbf{r})$ where $\kappa=\frac{1}{4} \operatorname{Re}(\alpha)$ and $\alpha$ is the particle polarizability, which depends on the particle's volume, shape and composition ${ }^{38}$. For a spherical particle with radius $R$ and refractive index $n_{p}$ immersed in a liquid with refractive index $n_{m}$, $\alpha=4 \pi n_{m}^{2} \varepsilon_{0} R^{3} \frac{n_{p}^{2}-n_{m}^{2}}{n_{p}^{2}+n_{m}^{2}}$. Inserting the full expression of the force and neglecting inertial effects in the motion of the particle ${ }^{29}$, Equation (4) simplifies as:

$$
\dot{\mathbf{r}}=\sqrt{2 D_{S E}} \mathbf{W}+\frac{\kappa}{\gamma} \nabla I,
$$

where $D_{S E}=\frac{k_{B} T}{\gamma}$ is the Stokes-Einstein's diffusion coefficient of the Brownian particle. Simulations of Brownian motion in the field of forces generated by a speckle pattern were therefore obtained by numerically solving Equation $(5)^{39}$. In all simulations, the particles are polystyrene beads $\left(n_{p}=1.59\right)$ in water $\left(n_{m}=1.33, \eta=\right.$ $0.001 \mathrm{Ns} / \mathrm{m}^{2}, T=300 \mathrm{~K}$ ). Due to the low scattering cross-section of the particles and their low concentration, we consider that the force field of the speckle pattern is not influenced by the particle. Even though we used a 2-dimensional model to simulate Brownian dynamics, our results can be readily extended to a 3-dimensional situation by considering a fully 3 -dimensional description of the speckle pattern ${ }^{24,39}$. In the presence of a hard boundary, an effective diffusion constant and an effective friction coefficient must be included in the model ${ }^{40}$, while for the case of low density suspensions of Brownian particles multiple reflections are very small, and can be safely neglected. In the presence of flow, we assumed laminar flow because of the low Reynolds numbers associated to microfluidc channel $\mathrm{s}^{29,40}$. Every particle trajectory used in the data presented from Figure 1 to 3 was calculated over a different realization of speckle field. Moreover, the initial position for the trajectory was randomly chosen within the speckle field. In Figure 4, instead, we used the same speckle field and initial position for every simulated particle. 
Mean square displacement calculation. The calculation of the MSD is performed according to $\operatorname{MSD}(\Delta t)=\left\langle\mathbf{r}^{2}(\Delta t)\right\rangle=\int \mathbf{r}^{2} P(\mathbf{r}, \Delta t) d^{2} \mathbf{r}$, where $P(\mathbf{r}, \Delta t)$ is the probability density function of finding a particle at position $\mathbf{r}$ at time $\Delta t^{1}$. In practice, each MSD curve was obtained by averaging over 500 different particle trajectories that were simulated over $100 \mathrm{~s}$ after waiting enough time for the particles to thermalize in the random optical potential given by the speckle pattern.

1. Barkai, E., Garini, Y. \& Metzler, R. Strange kinetics of single molecules in living cells. Phys. Today 65, 29-35 (2012).

2. Chandrasekhar, S. Brownian motion, dynamical friction, and stellar dynamics. Rev. Mod. Phys. 21, 383-388 (1949).

3. Goodman, J. W. Some fundamental properties of speckle. J. Opt. Soc. Am. 66, 1145 1150 (1976).

4. Mosk, A. P., Lagendijk, A., Lerosey, G. \& Fink, M. Controlling waves in space and time for imaging and focusing in complex media. Nature Photon. 6, 283-292 (2012).

5. Babic, D., Schmitt, C. \& Bechinger, C. Colloids as model systems for problems in statistical physics. Chaos 15, 026114 (2005)

6. Boiron, D. et al. Trapping and cooling cesium atoms in a speckle field. Eur. Phys. J. D 7, 373-377 (1999).

7. Shvedov, V. G. et al. Laser speckle field as a multiple particle trap. J. Opt. 12 124003 (2010).

8. Shvedov, V. G. et al. Selective trapping of multiple particles by volume speckle field. Opt. Express 18, 3137-3142 (2010).

9. Staforelli, J. P., Brito, J. M., Vera, E., Solano, P. \& Lencina, A. A clustered speckle approach to optical trapping. Opt. Commun. 283, 4722-4726 (2010).

10. Douglass, K. M., Sukhov, S. \& Dogariu, A. Superdiffusion in optically controlled active media. Nature Photon. 6, 834-837 (2012).

11. Čižmár, T., Mazilu, M. \& Dholakia, K. In situ wavefront correction and its application to micromanipulation. Nature Photon. 4, 388-394 (2010).

12. Dholakia, K. \& Čižmár, T. Shaping the future of optical manipulation. Nature Photon. 5, 335-342 (2011).

13. Lee, S. H. \& Grier, D. G. Giant colloidal diffusivity on corrugated optical vortices. Phys. Rev. Lett. 96, 190601 (2006).

14. Ricárdez-Vargas, I., Rodríguez-Montero, P., Ramos-García, R. \& VolkeSepúlveda, K. Modulated optical sieve for sorting of polydisperse microparticles. Appl. Phys. Lett. 88, 121116 (2006)

15. Demergis, V. \& Florin, E. L. High precision and continuous optical transport using a standing wave optical line trap. Opt. Express 19, 20833-20848 (2011).

16. Šiler, M., Cižmár, T. \& Zemánek, P. Speed enhancement of multi-particle chain in a traveling standing wave. Appl. Phys. Lett. 100, 051103 (2012).

17. McDonald, M. P., Spalding, G. C. \& Dholakia, K. Fractionation of polydispersed colloid with acousto-optically generated potential energy landscapes. Nature $\mathbf{4 2 6}$, 421-424 (2003).

18. Milne, G., Rhodes, D., McDonald, M. \& Dholakia, K. Microfluidic sorting in an optical lattice. Opt. Lett. 32, 1144-1146 (2007).

19. Xiao, K. \& Grier, D. G. Multidimensional optical fractionation of colloidal particles with holographic verification. Phys. Rev. Lett. 104, 028302 (2010)

20. De Groot, S. R. \& Mazur, P. Non-equilibrium thermodynamics (Dover, New York, 1984).

21. Feng, S., Kane, C., Lee, P. A. \& Stone, A. D. Correlation and fluctuations of coherent wave transmission through disordered media. Phys. Rev. Lett. 61, 834-837 (1988).

22. Freund, I., Rosenbluh, M. \& Feng, S. Memory effects in propagation of optical waves though disordered media. Phys. Rev. Lett. 61, 2328-2331 (1988).

23. Jonáš, A. \& Zemánek, P. Light at work: the use of optical forces for particle manipulation, sorting, and analysis. Electrophoresis 29, 4813-4851 (2008).

24. Goodman, J. W. Speckle Phenomena in Optics: Theory and Applications (Roberts and Co. Publishers, Greenwood Village, 2007).
25. Zhang, B., Zerubia, J. \& Olivo-Marin, J. C. Gaussian approximations of fluorescence microscope point-spread function models. Appl. Opt. 46, 1819-1829 (2007).

26. Ashkin, A. History of optical trapping and manipulation of small-neutral particle, atoms, and molecules. IEEE J. Sel. Top. Quant. El. 6, 841-846 (2000).

27. Nieminen, T. A. et al. Optical tweezers computational toolbox. J. Opt. A $\mathbf{9}$, S196-S203 (2007).

28. Borghese, F., Denti, P. \& Saija, R. Scattering from model nonspherical particles (Springer, Heidelberg, 2007).

29. Purcell, E. M. Life at low Reynolds numbers. Am. J. Phys. 45, 3-11 (1977).

30. McCann, L. I., Dykman, M. \& Golding, B. Thermally activated transitions in a bistable three-dimensional optical trap. Nature 402, 785-787 (1999).

31. Curry, N. et al. Direct determination of diffusion properties of random media from speckle contrast. Opt. Express 36, 3332-3334 (2011).

32. Bouchad, J. P. \& Georges, A. Anomalous diffusion in disordered media: statistical mechanisms, models and physical applications. Rep. Phys. 195, 127-293 (1990).

33. Rousselet, J., Salome, L., Ajdari, A. \& Prost, J. Directional motion of Brownian particles induced by a periodic asymmetric potential. Nature 370, 446-448 (1994).

34. Mikhael, J., Roth, J., Helden, L. \& Bechinger, C. Archimedean-like tilting on decagonal quasicrystalline surfaces. Nature 454, 501-504 (2008).

35. Thalhammer, G. et al. Combined acoustic and optical trapping. Biomed. Opt. Express 2, 2859-2870 (2011).

36. Ghielmetti, G. \& Aegerter, C. M. Scattered light fluorescence microscopy in three dimensions. Opt. Express 20, 3744-3752 (2012).

37. Volpe, G., Volpe, G. \& Petrov, D. Brownian motion in a nonhomogeneous force field and photonic force microscope. Phys. Rev. E 76, 061118 (2007).

38. Harada, Y. \& Asukura, T. Radiation forces on a dielectric sphere in the Rayleigh scattering regime. Opt. Commun. 124, 529-541 (1996).

39. Volpe, G. \& Volpe, G. Simulation of a Brownian particle in an optical trap. Am. J. Phys. 81, 224-230 (2013).

40. Happel, J. \& Brenner, H. Low Reynolds number hydrodynamics (Springer, New York, 1983).

\section{Acknowledgments}

The authors thank Andrew Griffiths and Patrick Tabeling for insightful discussions. Giovanni Volpe was partially supported by Marie Curie Career Integration Grant (MC-CIG) PCIG11 GA-2012-321726. Sylvain Gigan acknowledges funding from Agence Nationale de La Recherche (ANR-JCJC-ROCOCO), the City of Paris (Programme Emergence) and the European Research Council (under grant $\mathrm{N}^{\circ} 278025$ ).

\section{Author contributions}

G.V., G.V. and S.G. contributed to the original idea. G.V. and G.V. developed the theoretical model. G.V. (Giorgio Volpe) performed the numerical simulations. All authors discussed the results and contributed to the redaction of the manuscript.

\section{Additional information}

Supplementary information accompanies this paper at http://www.nature.com/ scientificreports

Competing financial interests: The authors declare no competing financial interests. How to cite this article: Volpe, G., Volpe, G. \& Gigan, S. Brownian Motion in a Speckle Light Field: Tunable Anomalous Diffusion and Selective Optical Manipulation. Sci. Rep. 4, 3936; DOI:10.1038/srep03936 (2014).

his work is licensed under a Creative Commons Attribution 3.0 Unported license To view a copy of this license, visit http://creativecommons.org/licenses/by/3.0 